\title{
Toll of vaccine hesitancy
}

Lancet Pub. Health https://doi.org/10.1016/S24682667(20)30010-4

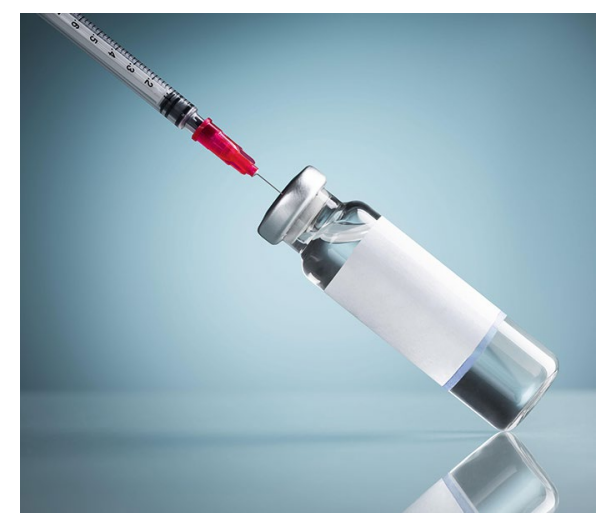

Credit: Andriy Popov / Alamy Stock Photo

In 2013, widely-reported concerns over possible adverse effects of the human papillomavirus (HPV) vaccine led to a fall in coverage of the vaccine in Japan. HPV vaccination had been introduced from 2010 for girls aged $12-16$, and $70 \%$ coverage was initially achieved, but after 2013, when the government suspended proactive recommendations, uptake fell to just $1 \%$ and remains low, despite no evidence of a causal link between the vaccine and the reported symptoms.

In a new study, teams from Cancer Council New South Wales (NSW) in Australia and Hokkaido University modelled the impact of Japan's HPV vaccine coverage drop. Using the Policy1-Cervix software model, the authors calculated that at least 24,600 additional cervical cancer cases and 5,000 deaths are expected to occur as a result of the crisis, compared to if uptake had remained at $70 \%$ after 2013 .

However, the authors show that if high vaccine coverage including 'catch-up' vaccination were implemented immediately, well over half of these additional cases and deaths could yet be prevented, and they point to Ireland and Denmark as examples of countries where strong support from government and other stakeholders successfully reversed HPV vaccine hesitancy.

The authors say that this is the first comprehensive attempt to quantify the implications of Japan's HPV vaccine crisis.

Jamie Horder

Published online: 17 April 2020

https://doi.org/10.1038/s41562-020-0874-1 